\author{
BARANETSKIJ YA.O. ${ }^{1}$, KALENYUK P.I. ${ }^{1}$, KOPACH M.I. ${ }^{2}$, SOlOMKO A.V. ${ }^{2}$
}

\title{
THE NONLOCAL BOUNDARY VALUE PROBLEM WITH PERTURBATIONS OF MIXED BOUNDARY CONDITIONS FOR AN ELLIPTIC EQUATION WITH CONSTANT COEFFICIENTS. II
}

\begin{abstract}
In this paper we continue to investigate the properties of the problem with nonlocal conditions, which are multipoint perturbations of mixed boundary conditions, started in the first part. In particular, we construct a generalized transform operator, which maps the solutions of the self-adjoint boundary-value problem with mixed boundary conditions to the solutions of the investigated multipoint problem. The system of root functions $V(L)$ of operator $L$ for multipoint problem is constructed. The conditions under which the system $V(L)$ is complete and minimal, and the conditions under which it is the Riesz basis are determined. In the case of an elliptic equation the conditions of existence and uniqueness of the solution for the problem are established.

Key words and phrases: differential equation with partial derivatives, root functions, Fourier method, method of transform operators, Riesz basis.
\end{abstract}

\footnotetext{
${ }^{1}$ Lviv Polytechnic National University, 12 Bandera str., 79013, Lviv, Ukraine

${ }^{2}$ Vasyl Stefanyk Precarpathian National University, 57 Shevchenka str., 76018, Ivano-Frankivsk, Ukraine

E-mail: baryaromeukr. net (Baranetskij Ya.O.), kalenyuk@lp. edu . ua (Kalenyuk P.I.), kopachm2009@gmail.com (Kopach M.I.), ansolvasegmail.com (Solomko A.V.)
}

\section{INTRODUCTION AND MAIN RESULTS}

In the papers [1-5] by the methods of the theory of transformation operators (see [12]), we studied nonself-adjoint problems with a multipoint spectrum and an infinite number of root functions (see [10]). In the one-dimensional case such problems are generated by regular but not strongly regular Birkhoff conditions (see [11]). For equations containing involution, multipoint problems were studied in the works [5-7]. In this paper we continue the study of the problem for an elliptic equations with constant coefficients with mixed conditions initiated in $[1,7,8]$.

For our investigation we will use the following notations. Let $G:=\left\{x:=\left(x_{1}, x_{2}\right) \in \mathbb{R}^{2}\right.$ : $\left.0<x_{1}, x_{2}<1\right\}, D_{1}, D_{2}$ be the operators of differentiation by the variables $x_{1}, x_{2}$ respectively, $W_{2}^{2 n}(G)$ be a Sobolev space with the following scalar product and norm respectively:

$$
\begin{aligned}
(u ; v)_{W_{2}^{2 n}(G)} & :=(u ; v)_{L_{2}(G)}+\left(D_{1}^{2 n} u ; D_{1}^{2 n} v\right)_{L_{2}(G)}+\left(D_{2}^{2 n} u ; D_{2}^{2 n} v\right)_{L_{2}(G)}, \\
\|u\|_{W_{2}^{2 n}(G)}^{2 n} & :=(u ; u)_{W_{2}^{2 n}(G)} \\
W_{2}^{2 n}(0,1) & :=\left\{y \in A C[0,1]: y^{(r)} \in C[0,1], r=1,2, \ldots, 2 n-1, y^{(2 n)} \in L_{2}(0,1)\right\}, \\
L_{s, 2}(0,1) & :=\left\{y(t) \in L_{2}(0,1): y(t)=(-1)^{s} y(1-t)\right\}, s \in\{0,1\},
\end{aligned}
$$

$\mathrm{y} \Delta \mathrm{K} 517.927 .5,517.984 .5$

2010 Mathematics Subject Classification: 34G10, 34K10, 34K30, 34L10. 
$\left[L_{2}(0,1)\right]$ be a set of linear and continuous operators given in the space $L_{2}(0,1)$.

Let us consider the multipoint problem

$$
\begin{gathered}
L(D) u:=\sum_{p=0}^{n} a_{p} D_{1}^{2 p} D_{2}^{2 n-2 p} u=f(x), x \in G, \\
\begin{cases}\ell_{s, 1} u \quad:=\left.D_{1}^{2 s-2} u\right|_{x_{1}=0}+\left.D_{1}^{2 s-2} u\right|_{x_{1}=1}+\ell_{s}^{1} u=0, s=1,2, \ldots, n, \\
\ell_{n+s, 1} u \quad:=\left.D_{1}^{2 s-2} u\right|_{x_{1}=0}-\left.D_{1}^{2 s-2} u\right|_{x_{1}=1}=0, s=1,2, \ldots, n, \\
\ell_{s, 2} u \quad:=\left.D_{2}^{2 s-2} u\right|_{x_{2}=0}+\left.D_{2}^{2 s-2} u\right|_{x_{2}=1}=0, s=1,2, \ldots, n, \\
\ell_{n+s, 2} u:=\left.D_{2}^{2 s-1} u\right|_{x_{2}=0}+\left.D_{2}^{2 s-1} u\right|_{x_{2}=1}+\ell_{s}^{2} u=0, s=1,2, \ldots, n, \\
\ell_{s}^{j} u \quad:=\left.\sum_{q=0}^{k_{s, j}} k_{j} b_{q, r, s, j} D_{j}^{q} u(x)\right|_{x_{j}=x_{r, j},} s=1,2, \ldots, n,\end{cases}
\end{gathered}
$$

where $0=x_{1, j}<x_{2, j}<\ldots<x_{k_{j, j}}=1, a_{p}, b_{q, r, s, j} \in \mathbb{R}, k_{s, j}<2 n, k \in \mathbb{N}, s=1,2, \ldots, n$, $p=0,1, \ldots, n, j=1,2$.

Let $L: L_{2}(G) \rightarrow L_{2}(G)$ be the operator of the problem (1)-(2), $L u:=L(D) u, u \in D(L)$, $D(L):=\left\{u \in W_{2}^{2 n}(G): \ell_{s, j} u=0, s=1,2, \ldots, 2 n, j=1,2\right\}$.

Let us consider the following assumptions and theorems, that are necessary for further investigation.

Assumption $P_{1}: b_{q, r, s, j}=(-1)^{q+j} b_{q, k_{j}-r, s, j}, x_{r, j}=1-x_{k_{j}-r, j}, q=0,1, \ldots, k_{s, j}, r=0,1, \ldots, k_{j}$, $s=1,2, \ldots, n, j=1,2$.

Assumption $P_{2}$ : there exists a positive number $C_{1}$ such that the inequality

$$
C_{1}|\mu|^{2 n} \leq\left|\sum_{p=0}^{n} a_{p} \mu_{1}^{p} \mu_{2}^{n-p}\right|
$$

holds for $\mu:=\left(\mu_{1}, \mu_{2}\right) \in \mathbb{R}^{2},|\mu|^{2}:=\left|\mu_{1}\right|^{2}+\left|\mu_{2}\right|^{2} \rightarrow \infty$.

Assumption $P_{3}: k_{s, 1} \leq 2 s-2, k_{s, 2} \leq 2 s-1, s=1,2, \ldots, n$.

Theorem 1. Let Assumption $P_{1}$ holds. Then, the operator $L$ has a set of eigenvalues

$$
\sigma:=\left\{\lambda_{k, m}=(-1)^{n} \sum_{p=0}^{n} a_{p} \mu_{k, 1}^{p} \mu_{m, 2}^{n-p}, \mu_{k, 1}=\pi^{2} k^{2}, \mu_{m, 2}=\pi^{2}(2 m-1)^{2}, k, m \in \mathbb{N}\right\},
$$

and a system $V(L)$ of root functions, which is complete and minimal in the space $L_{2}(G)$.

Let Assumptions $P_{1}-P_{3}$ hold. Then, the operator $L$ has the system $V(L)$, which is the Riesz basis of the space $L_{2}(G)$.

Theorem 2. Let Assumptions $P_{1}-P_{3}$ hold. Then for an arbitrary function $f \in L_{2}(G)$ there exists a unique solution $u \in W_{2}^{2 n}(G)$ of problem (1)-(2).

Our research is structured as follows. In Section 2 we investigate the properties of the problem with self-adjoint boundary conditions. In Section 3 we study the spectral properties for nonlocal problem with nonself-adjoint boundary conditions. In Section 4 we construct a commutative group of transformation operators. Using spectral properties of multipoint problem and conditions for completeness the basis properties of the systems of eigenfunctions are established in Section 5. In Section 6 the main theorems are proved. 


\section{THE SELF-AJOINT PROBLEM}

Let us consider for equation

$$
-z^{(2)}(t)=\mu z(t), \quad t \in(0,1), \mu \in \mathbb{C},
$$

the problem with boundary conditions

$$
z^{(r)}(0)+z^{(r)}(1)=0, \quad r=0,1
$$

Let $B_{0}: L_{2}(0,1) \rightarrow L_{2}(0,1)$ be the operator of problem (4)-(5):

$$
\begin{aligned}
& B_{0} z(t):=-z^{(2)}(t), z(t) \in D\left(B_{0}\right), \\
& D\left(B_{0}\right):=\left\{z \in W_{2}^{2}(0,1): z^{(r)}(0)+z^{(r)}(1)=0, r=0,1\right\}, \\
& T_{2}:=\left\{\tau_{r, m, 2}(t) \in L_{2}(0,1): \tau_{0, m, 2}(t):=\sqrt{2} \sin \pi(2 m-1) t,\right. \\
& \left.\tau_{1, m, 2}(t):=\sqrt{2} \cos \pi(2 m-1) t, m \in \mathbb{N}, r=0,1\right\}, \\
& T_{j, 2}:=\left\{\tau_{j, m, 2}(t) \in L_{j, 2}(0,1): m \in \mathbb{N}\right\}, j=0,1 .
\end{aligned}
$$

Lemma 1. The operator $B_{0}$ has a point spectrum

$$
\sigma\left(B_{0}\right):=\left\{\mu_{m, 2} \in \mathbb{R}: \mu_{m, 2}=\pi^{2}(2 m-1)^{2}, m \in \mathbb{N}\right\}
$$

and system of eigenfunctions $T_{2}$.

Proof. A direct substitution proves that the elements of system $T_{2}$ are the eigenfunctions of operator $B_{0}$, which correspond to the eigenvalues $\sigma\left(B_{0}\right)$.

Taking into account, that the subsystem of eigenfunctions $T_{j, 2}$ of the operator $B_{0}$ is an orthonormal base of space $L_{j, 2}(0,1), j=0,1$, we obtain the statement of lemma.

We consider the spectral problem

$$
\begin{gathered}
L(D) u:=\sum_{p=0}^{n} a_{p} D_{1}^{2 p} D_{2}^{2 n-2 p} u=\lambda u(x), x \in G, \lambda \in \mathbb{C}, \\
\begin{cases}\ell_{0, s, 1} u \quad:=\left.D_{1}^{2 s-2} u\right|_{x_{1}=0}+\left.D_{1}^{2 s-2} u\right|_{x_{1}=1}=0 \\
\ell_{0, n+s, 1} u & :=\left.D_{1}^{2 s-2} u\right|_{x_{1}=0}-\left.D_{1}^{2 s-2} u\right|_{x_{1}=1}=0 \\
\ell_{0, s, 2} u & :=\left.D_{2}^{2 s-2} u\right|_{x_{2}=0}+\left.D_{2}^{2 s-2} u\right|_{x_{2}=1}=0 \\
\ell_{0, n+s, 2} u & :=\left.D_{2}^{2 s-1} u\right|_{x_{2}=0}+\left.D_{2}^{2 s-1} u\right|_{x_{2}=1}=0, s=1,2, \ldots, n .\end{cases}
\end{gathered}
$$

Let $L_{0}: L_{2}(G) \rightarrow L_{2}(G)$ be the operator of the problem (6)-(7):

$$
\begin{aligned}
L_{0} u & :=L(D) u, u \in D\left(L_{0}\right), D\left(L_{0}\right):=\left\{u \in W_{2}^{2 n}(G): \ell_{0, r, j} u=0, r=1,2, \ldots, 2 n, j=1,2\right\}, \\
T_{1} & :=\left\{\tau_{s, k, 1}\left(x_{1}\right) \in L_{2}(0,1): \tau_{s, k, 1}\left(x_{1}\right):=\sqrt{2} \sin \pi(2 k-s) x_{1}, k=1,2, \ldots, s=0,1\right\}, \\
V\left(L_{0}\right) & :=\left\{v_{r, s, k, m}\left(x, L_{0}\right) \in L_{2}(G): v_{r, s, k, m}\left(x, L_{0}\right):=\tau_{r, k, 1}\left(x_{1}\right) \tau_{s, m, 2}\left(x_{2}\right), r, s=0,1, m, k=1,2, \ldots\right\} .
\end{aligned}
$$


Lemma 2. The operator $L_{0}$ has eigenvalues (3) and a system of eigenfunctions $V\left(L_{0}\right)$.

Proof. By direct substitution we obtain that $v_{r, s, k, m}\left(x, L_{0}\right) \in D\left(L_{0}\right)$ and

$$
\begin{aligned}
L_{0} v_{r, s, k, m}\left(x, L_{0}\right) & =\lambda_{k, m} v_{r, s, k, m}\left(x, L_{0}\right), \\
\lambda_{k, m} & =(-1)^{n} \pi^{2 n} \sum_{p=0}^{n} a_{p} k^{2 p}(2 m-1)^{2 n-2 p}, \quad k, m \in \mathbb{N} .
\end{aligned}
$$

Therefore, the set of eigenvalues (3) for the operator $L_{0}$ corresponds the system of eigenfunctions $V\left(L_{0}\right)$.

Theorem 3. Let Assumption $P_{2}$ holds. Then for any function $f \in L_{2}(G)$ there exists a unique solution $u \in W_{2}^{2 n}(G)$ of the problem (6)-(7).

Proof. Let us expand the functions $f, u \in L_{2}(G)$ as a series by the system $V\left(L_{0}\right)$ :

$$
\begin{aligned}
& f=\sum_{r, s, k, m} f_{r, s, k, m} v_{r, s, k, m}\left(x, L_{0}\right), \\
& u=\sum_{r, s, k, m} u_{r, s, k, m} v_{r, s, k, m}\left(x, L_{0}\right) .
\end{aligned}
$$

Substituting these functions into the equation (1), we obtain

$$
u_{r, s, k, m}=\lambda_{k, m}^{-1} f_{r, s, k, m}, r, s \in\{0,1\}, k, m \in \mathbb{N} .
$$

Consider the ratio

$$
D_{1}^{2 p} D_{2}^{2 n-2 p} u=(-1)^{n} \sum_{r, s, k, m} \mu_{k, 1}^{p} \mu_{m, 2}^{n-p} \lambda_{k, m}^{-1} f_{r, s, k, m} v_{r, s, k, m}\left(x, L_{0}\right), p=0,1, \ldots, n .
$$

Taking into account Assumption $P_{2}$ for some $C_{2}>0$, we obtain

$$
\begin{aligned}
\left|\mu_{k, 1}^{p} \mu_{m, 2}^{n-p} \lambda_{k, m}^{-1}\right| & \leq C_{2}, p=0,1, \ldots, n, \\
\left\|D_{1}^{2 p} D_{2}^{2 n-2 p} u\right\|_{L_{2}(G)} & \leq C_{2}\|f\|_{L_{2}(G)}, p=0,1, \ldots, n, \\
\|u\|_{L_{2}(G)} & \leq C_{2}\|f\|_{L_{2}(G)} .
\end{aligned}
$$

Therefore, $u \in W_{2}^{2 n}(G)$. Theorem is proved.

For fixed $k \in \mathbb{N}, s \in\{0,1\}$, we consider the solutions of the problem (6)-(7) as a product

$$
u(x):=z\left(x_{2}\right) \tau_{s, k, 1}\left(x_{1}\right) .
$$

To determine the unknown function $z\left(x_{2}\right)$ we obtain the following eigenvalues problem

$$
\begin{aligned}
& \sum_{p=0}^{n}(-1)^{p} a_{p} \mu_{k, 1}^{p} z^{(2 n-2 p)}\left(x_{2}\right)=\lambda z\left(x_{2}\right), x_{2} \in(0,1), \lambda \in \mathbb{C}, \\
& \begin{cases}l_{0, s, 2} z & :=z^{(2 s-2)}(0)+z^{(2 s-2)}(1)=0, \\
l_{0, n+s, 2} z & :=z^{(2 s-1)}(0)+z^{(2 s-1)}(1)=0, s=1,2, \ldots, n .\end{cases}
\end{aligned}
$$


Let $L_{0, k}: L_{2}(0,1) \rightarrow L_{2}(0,1)$ be the operator of the problem (9)-(10)

$$
\begin{gathered}
L_{0, k} z:=\sum_{p=0}^{n}(-1)^{p} a_{p} \mu_{k, 1}^{p} z^{(2 n-2 p)}\left(x_{2}\right), z \in D\left(L_{0, k}\right), \\
D\left(L_{0, k}\right):=\left\{z \in W_{2}^{2 n}(0,1): l_{0, r, 2} z=0, \quad r=1,2, \ldots, 2 n\right\} .
\end{gathered}
$$

The roots $\omega_{r, k}(\lambda)$ of the equation

$$
\sum_{p=0}^{n}(-1)^{p} a_{p} \omega^{2 n-2 p} \mu_{k, 1}^{p}=\lambda
$$

which is characteristic for equation (9), are chosen so that

$\operatorname{Re} \omega_{n, k}(\lambda) \leq \operatorname{Re} \omega_{n-1, k}(\lambda) \leq \ldots \leq \operatorname{Re} \omega_{1, k}(\lambda) \leq 0, \omega_{n+q, k}(\lambda)=-\omega_{n, k}(\lambda), q=1,2, \ldots, n$

Let us determine the functions

$$
\left\{\begin{array}{l}
z_{0, q, k}\left(x_{2}, \lambda\right):=\frac{1}{2}\left(\exp \omega_{q, k}(\lambda) x_{2}+\exp \omega_{q, k}(\lambda)\left(1-x_{2}\right)\right) \in L_{0,2}(0,1), \quad q=1, \ldots, n, \\
z_{0, n+q, k}\left(x_{2}, \lambda\right):=\frac{1}{2}\left(\exp \omega_{q, k}(\lambda) x_{2}-\exp \omega_{q, k}(\lambda)\left(1-x_{2}\right)\right) \in L_{1,2}(0,1), \quad q=1, \ldots, n
\end{array}\right.
$$

Substituting the general solution

$$
z\left(x_{2}\right)=\sum_{r=1}^{2 n} c_{r} z_{0, r, k}\left(x_{2}, \lambda\right)
$$

of the equation (9) into boundary conditions (10), we obtain the equation to determine the eigenvalues for $L_{0, k}$

$$
\Delta(\lambda, k)=\operatorname{det}\left(l_{0, s, 2} z_{0, r, k}\left(x_{2}, \lambda\right)\right)_{r, s=1}^{2 n}=0 .
$$

Taking into account the ratio $z_{0, p n+q, k}\left(x_{2}, \lambda\right) \in L_{p, 2}(0,1), l_{0, p n+j, 2} \in W_{p}^{*}, p \in\{0,1\}$, we obtain

$$
\begin{aligned}
l_{0, n+j, 2} z_{0, q, k}\left(x_{2}, \lambda\right) & =0, j, q=1,2, \ldots, n, \\
l_{0, j, 2} z_{0, n+q, k}\left(x_{2}, \lambda\right) & =0, j, q=1,2, \ldots, n, \\
\Delta(\lambda, k) & =\Delta_{0}(\lambda, k) \Delta_{1}(\lambda, k), \\
\Delta_{p}(\lambda, k) & =\operatorname{det}\left(l_{0, p n+j, 2} z_{0, p n+q, k}\left(x_{2}, \lambda\right)\right)_{j, q=1}^{n}, p=0,1, \\
\Delta(\lambda, k)=\prod_{q=1}^{n} \omega_{q}(\lambda) & \left(1+e^{\omega_{q}(\lambda)}\right)^{2} \prod_{1 \leq j<q \leq n}\left(\omega_{j}(\lambda)-\omega_{q}(\lambda)\right)^{2}=0 .
\end{aligned}
$$

Let $\omega_{1, m, k}=\imath \pi(2 m-1), \imath:=\sqrt{-1}$ are the roots of equation (12) and $\omega_{q, m, k}=\omega_{q}\left(\lambda_{m, k}\right)$, $q=2,3, \ldots, n, m=1,2, \ldots$. By direct calculations we obtain that the operator $L_{0, k}$ has the system of eigenfunctions

$$
V\left(L_{0, k}\right):=\left\{v_{s, m}\left(x_{2}, L_{0, k}\right) \in L_{2}(0,1): v_{s, m}\left(x_{2}, L_{0, k}\right):=\tau_{s, m, 2}\left(x_{2}\right), s=0,1, m=1,2, \ldots\right\}
$$

and the set of eigenvalues $\sigma_{k}:=\left\{\lambda_{k, m} \in \sigma: m \in \mathbb{N}\right\}$. 


\section{THE NONSELF-AJOINT PROBLEM}

Let us consider the spectral problem

$$
\left\{\begin{array}{l}
-z^{(2)}(t)=\mu z(t), \mu \in \mathbb{C}, t \in(0,1), \\
l_{1} z:=z(0)+z(1)=0 \\
l_{2} z:=z^{(1)}(0)+z^{(1)}(1)+l_{2}^{1} z=0,
\end{array}\right.
$$

where

$$
l_{2}^{1} z:=b\left(z^{(1)}(0)-z^{(1)}(1)\right), b \in \mathbb{R} .
$$

Let $B: L_{2}(0,1) \rightarrow L_{2}(0,1)$ be the operator of the problem (13)-(14) and $V(B)$ the system of root functions for operator $B$.

Taking into account the results of the papers [1,2], we define eigenfunctions and attach functions of the operators $B$ by formulas

$$
\begin{aligned}
& v_{1, m}(t, B)=\tau_{1, m, 2}(t), \\
& v_{0, m}(t, B)=(1+b(2 t-1)) \tau_{0, m, 2}(t), m=1,2, \ldots .
\end{aligned}
$$

Therefore, the operator $B$ has the system $V(B)$ of root functions, which are related by ratio

$$
B v_{0, m}(t, B)=\mu_{m, 2} v_{0, m}(t, B)+\xi_{m} v_{1, m}(t, B),
$$

where $\xi_{m}=4 b \pi(2 m-1), m=1,2, \ldots$

Taking into account the results of the paper [2], we obtain the following statement.

Lemma 3. The operator $B$ has the point spectrum $\sigma\left(B_{0}\right)$ and the system of root functions $V(B)$, which is the Riesz basis of the space $L_{2}(0,1)$.

We consider the solutions of the spectral problem (6), (2) as a product (8). To determine the unknown function $z\left(x_{2}\right)$ we obtain for the equation (9) the eigenvalues problem with the conditions

$$
\left\{\begin{array}{l}
l_{s, 2} z:=z^{(2 s-2)}(0)+z^{(2 s-2)}(1)=0, \\
l_{n+s, 2} z:=z^{(2 s-1)}(0)+z^{(2 s-1)}(1)+l_{n+s} z=0, s=1,2, \ldots, n,
\end{array}\right.
$$

where

$$
l_{n+s} z:=\sum_{q=0}^{k_{s, 2}} \sum_{r=0}^{k_{2}} b_{q, r, s, 2} z^{(q)}\left(x_{r, 2}\right), s=1,2, \ldots, n .
$$

Let $L_{k}$ be the operator of the problem (9), (15)-(16):

$$
\begin{aligned}
L_{k} z & :=\sum_{p=0}^{n}(-1)^{p} a_{p} \mu_{k, 1}^{p} z^{(2 n-2 p)}\left(x_{2}\right), z \in D\left(L_{k}\right), \\
D\left(L_{k}\right) & :=\left\{z \in W_{2}^{2 n}(0,1): l_{r, 2} z=0, r=1,2, \ldots, 2 n\right\} .
\end{aligned}
$$


Lemma 4. Let Assumption $P_{1}$ holds. Then the eigenvalues of the operators $L_{0, k}$ and $L_{k}$ coincide.

Proof. Substituting the general solution (11) of the equation (9) into boundary conditions (10), we obtain the equation to determine the eigenvalues of the operator $L_{k}$

$$
\Delta^{1}(\lambda, k)=\operatorname{det}\left(l_{s, 2} z_{0, p, k}\left(x_{2}, \lambda\right)\right)_{p, s=1}^{2 n}=0 .
$$

Taking into account the relations

$$
z_{0, r n+q, k}\left(x_{2}, \lambda\right) \in L_{r, 2}(0,1), \ell_{0, s n+j, 2} \in W_{s}^{*}, l_{n+j}^{1} \in W_{0}^{*}, s, r \in\{0,1\}, j \in\{1,2, \ldots, n\},
$$

we obtain

$$
\begin{aligned}
l_{j, 2} z_{0, q, k}\left(x_{2}, \lambda\right) & =\omega_{q}^{2 j-2}(\lambda)\left(1+e^{\omega_{q}(\lambda)}\right), \\
l_{n+j, 2} z_{0, n+q, k}\left(x_{2}, \lambda\right) & =\omega_{q}(\lambda)^{2 j-1}\left(1+e^{\omega_{q}(\lambda)}\right), \\
l_{j, 2} z_{0, n+q, k}\left(x_{2}, \lambda\right) & =l_{0, j, 2} z_{0, n+q, k}\left(x_{2}, \lambda\right)=0, \\
l_{n+j, 2} z_{0, n+q, k}\left(x_{2}, \lambda\right) & =l_{0, n+j, 2} z_{0, n+q, k}\left(x_{2}, \lambda\right), \\
l_{j, 2} z_{0, q, k}\left(x_{2}, \lambda\right) & =l_{j, 2,2} z_{0, q, k}\left(x_{2}, \lambda\right), \quad q=1,2, \ldots, n, \\
\Delta(\lambda, k) & =\Delta_{0}(\lambda, k) \Delta_{1}(\lambda, k), \\
\Delta_{s}(\lambda, k) & =\operatorname{det}\left(l_{0, s n+j, 2} z_{0, s n+q, k}\left(x_{2}, \lambda\right)\right)_{j, q=1}^{n}, \quad s=0,1,
\end{aligned}
$$

and

$$
\Delta^{1}(\lambda, k)=\prod_{q=1}^{n} \omega_{q}(\lambda)\left(1+e^{\omega_{q}(\lambda)}\right)^{2} \prod_{1 \leq j<q \leq n}\left(\omega_{j}(\lambda)-\omega_{q}(\lambda)\right)^{2}=0 .
$$

Therefore $\Delta^{1}(\lambda, k) \equiv \Delta(\lambda, k)$. The lemma is proved.

Let us consider the boundary-value problem for the equation (9)

$$
\left\{\begin{array}{l}
l_{1, s, 2} z:=z^{(2 s-2)}(0)+z^{(2 s-2)}(1)=0, s=1,2, \ldots, n, \\
l_{1, n+s, 2} z:=z^{(2 s-1)}(0)+z^{(2 s-1)}(1)=0, j \neq s, s=1,2, \ldots, n, \\
l_{1, n+j, 2} z:=z^{(2 j-1)}(0)+z^{(2 j-1)}(1)+\ell_{n+j}^{1} z=0,
\end{array}\right.
$$

where

$$
l_{n+j}^{1} z:=b_{j}\left(z^{(2 j-1)}(0)-z^{(2 j-1)}(1)\right)=0, \quad b_{j} \in \mathbb{R} .
$$

Let $L_{1, j, k}: L_{2}(0,1) \rightarrow L_{2}(0,1)$ be the operator of the problem (9), (17)-(18)

$$
\begin{aligned}
L_{1, j, k} z & :=\sum_{p=0}^{n}(-1)^{p} a_{p} \mu_{k, 1}^{p} z^{(2 n-2 p)}\left(x_{2}\right), z \in D\left(L_{1, j, k}\right), \\
D\left(L_{1, j, k}\right): & =\left\{z \in W_{2}^{2 n}(0,1): l_{1, r, 2} z=0, \quad r=1,2, \ldots, 2 n\right\} .
\end{aligned}
$$

We determine the system of functions

$$
\begin{gathered}
z_{n+1, m, k}\left(x_{2}\right):=\frac{1}{2}\left(1-2 x_{2}\right) \sin \rho_{m, 2} x_{2}, \\
z_{n+q, m, k}\left(x_{2}\right):=\frac{1}{2}\left(1+e^{\omega_{q, m, k}}\right)^{-1}\left(e^{\omega_{q, m, k} x_{2}}-e^{\omega_{q, m, k}\left(1-x_{2}\right)}\right), \quad q=2,3, \ldots, n,
\end{gathered}
$$


and a square matrix of order $n$, whose elements are defined as follows. $j$-th row is determined by the functions (19), (20) and elements of other rows are determined by numbers

$$
\begin{aligned}
& \vartheta_{1, r, m, k}=\rho_{m, 2}^{1-2 r} l_{1, n+r, 2} z_{n+1, m, k}\left(x_{2}\right)=(-1)^{r-1}, \\
& \vartheta_{q, r, m, k}=\rho_{m, 2}^{1-2 r} l_{1, n+r, 2} z_{n+q, m, k}\left(x_{2}\right)=\omega_{q, m, k^{\prime}}^{2 r-1}
\end{aligned}
$$

where $q=2,3, \ldots, n, r \neq j, \quad r=1,2, \ldots, n$.

We denote the determinant of the resulting matrix by $z_{j, m, k}\left(x_{2}\right), m=1,2, \ldots$.

Let $\Delta_{j, q, m, k}:=\operatorname{det}\left(\vartheta_{s, r, m, k}\right)_{r, s=j, s \neq q}^{r \neq j}$. Then $z_{j, m, k}\left(x_{2}\right)=\sum_{q=1}^{n} \Delta_{j, q, m, k} z_{n+q, m, k}\left(x_{2}\right)$.

Remark 1. For any fixed $k \in \mathbb{N}$ and $m \rightarrow \infty$ we obtain the relations

$$
\delta_{1, r, m, k}=\vartheta_{1, r, m, k} \rho_{m, 2}^{-1}=\imath, \quad \delta_{q, r, m, k}=\vartheta_{q, r, m, k} \rho_{m, 2}^{-1}=\varepsilon_{q}\left(1+O(m)^{-1}\right),
$$

where $\varepsilon_{q}$ are the roots of equation $(-1)^{n} \varepsilon^{2 n}=1, \operatorname{Im} \varepsilon_{q}<0, q=2,3, \ldots, n$.

Substituting the function $z_{j, m, k}\left(x_{2}\right)$ into boundary conditions (17)-(18), we obtain the equalities

$$
\begin{aligned}
l_{1, s, 2} z_{j, m, k} & =0, s \neq n+j, s=1,2, \ldots, 2 n, \quad m=1,2, \ldots, \\
l_{1, n+j, 2} z_{j, m, k}\left(x_{2}\right) & :=c_{j, m, k \prime} \\
c_{j, m, k} & =\rho_{m, 2}^{2 j-1} Z_{m, k} \prod_{q=1}^{n} \omega_{q, m, k}, \quad m=1,2, \ldots,
\end{aligned}
$$

where $Z_{m, k}$ is the Vandermonde determinant of order $n$, which is constructed by numbers $\delta_{q, r, m, k}^{2} q=1,2, \ldots, n$.

Remark 2. For an arbitrary $k \in \mathbb{N}$ the number sequence $\left\{Z_{m, k}\right\}_{m=1}^{\infty}$ as $m \rightarrow \infty$ converges to the Vandermonde determinant $Z_{n}\left(\varepsilon_{1}^{2}, \ldots, \varepsilon_{n}^{2}\right)$, which is constructed by numbers $\varepsilon_{1}^{2}, \ldots, \varepsilon_{n}^{2}$.

In addition, the sequence $\left\{\delta_{q, r, m, k}\right\}_{m=1}^{\infty}$ converges to $\varepsilon_{q}, q=1,2, \ldots, n$.

Thus, there are positive numbers $C_{3}, C_{4}$ such that the following inequality holds

$$
0<C_{3} \leq\left|c_{j, m, k}\right|^{-1} \rho_{m, 2}^{2 j-1} \leq C_{4}<\infty, j \in\{1,2, \ldots, n\}, m=1,2, \ldots
$$

We determine the function $z_{1, j, m, k}\left(x_{2}\right)$ such that the following inequality holds

$$
z_{1, j, m, k}\left(x_{2}\right)=z_{n+1, m, k}\left(x_{2}\right)+\sum_{q=2}^{n} \Delta_{j, 1, m, k}^{-1} \Delta_{j, q, m, k} z_{n+q, m, k}\left(x_{2}\right) .
$$

Therefore,

$$
\begin{gathered}
z_{1, j, m, k}\left(x_{2}\right)=\Delta_{j, 1, m, k}^{-1} z_{j, m, k}\left(x_{2}\right), \\
\ell_{1, n+j} z_{1, j, m, k}\left(x_{2}\right):=\chi_{j, m, k}, \quad \chi_{j, m, k}=\Delta_{j, 1, m, k}^{-1} z_{m, k} \rho_{m, 2}^{2 j-1} \prod_{q=1}^{n} \omega_{q, m, k}, m=1,2, \ldots
\end{gathered}
$$

By substituting into boundary conditions (17)-(18) we conclude that the operator $L_{1, j, k}$ has eigenfunctions

$$
v_{1, m}\left(x_{2}, L_{1, j, k}\right):=\tau_{1, m, 2}\left(x_{2}\right), \quad m=1,2, \ldots
$$


The root function $v_{0, m}\left(x_{2}, L_{1, j, k}\right)$ of operator $L_{1, j, k}$ is determined by the sum

$$
v_{0, m}\left(x_{2}, L_{1, j, k}\right):=\tau_{0, m, 2}\left(x_{2}\right)+\eta_{j, m, k} z_{1, j, m, k}\left(x_{2}\right), \quad m=1,2, \ldots
$$

To determine the unknown parameters $\eta_{j, m, k}$ we substitute the expression (24) into boundary conditions (17)-(18).

Taking into account the formula (22), we obtain

$$
\eta_{j, m, k}=-l_{n+j}^{1} \tau_{0, m, 2}\left(l_{1, n+j, 2} z_{1, j, m, k}\right)^{-1}, \quad m=1,2, \ldots
$$

From the definition of the determinant $\Delta_{j, 1, m, k}$ we have inequality $\left|\Delta_{j, 1, m, k}^{-1}\right| \leq C_{5}$.

Therefore, taking into account the inequality $\left|l_{n+j}^{1} \tau_{0, m, 2}\right| \leq C_{6} b_{j} \rho_{m, 2}^{2 j-1}$ and the estimates (21), we obtain the relations

$$
\left|\eta_{j, m, k}\right| \leq C_{7}, \quad j \in\{1,2, \ldots, n\}, m \in \mathbb{N} .
$$

Thus, the operator $L_{1, j, k}$ has the system of root functions (23)-(24).

Let us consider the operator $B_{j, k}: L_{2}(0,1) \rightarrow L_{2}(0,1)$, which has a point spectrum $\sigma\left(B_{0}\right)$ and the system of root functions

$$
\begin{aligned}
V\left(B_{j, k}\right):=\left\{v_{r, m}\left(x_{2}, B_{j, k}\right) \in L_{2}(0,1):\right. & v_{1, m}\left(x_{2}, B_{j, k}\right):=\tau_{1, m, 2}\left(x_{2}\right), \\
& \left.v_{0, m}\left(x_{2}, B_{j, k}\right):=\left(1+\eta_{j, m, k}\left(2 x_{2}-1\right)\right) \tau_{0, m, 2}\left(x_{2}\right), \quad m=1,2, \ldots\right\} .
\end{aligned}
$$

Lemma 5. The system of functions $V\left(B_{j, k}\right)$ is the Riesz basis in the space $L_{2}(0,1)$.

Proof. From the inequality (25) we obtain that the system $V\left(B_{j, k}\right)$ is Bessel (see [10]). Therefore, the operator $R\left(B_{j, k}\right) \tau_{r, m, 2}\left(x_{2}\right):=v_{r, m}\left(x_{2}, B_{j, k}\right), r=0,1, m=1,2, \ldots$, is continuous in $L_{2}(0,1)$.

In addition, the operator $S\left(B_{j, k}\right):=R\left(B_{j, k}\right)-E$ is continuous in the space $L_{2}(0,1)$.

Taking into account the definition of functions in $V\left(B_{j, k}\right)$ we obtain

$$
S\left(B_{j, k}\right): L_{1,2}(0,1) \rightarrow 0, \quad S\left(B_{j, k}\right): L_{0,2}(0,1) \rightarrow L_{1,2}(0,1) .
$$

Thus, $S^{2}\left(B_{j, k}\right)$ and $R^{-1}\left(B_{j, k}\right):=E-R\left(B_{j, k}\right) \in\left[L_{2}(0,1)\right]$.

Therefore, from the Bari theorem (see [10]) the system $V\left(B_{j, k}\right)$ is the Riesz basis in the space $L_{2}(0,1)$.

Lemma 6. Let Assumption $P_{1}$ holds. Then the operator $L_{1, j, k}$ has the system of root functions $V\left(L_{1, j, k}\right)$, which is the Riesz basis in the space $L_{2}(0,1)$.

Proof. The system of functions $V\left(L_{1, j, k}\right)$ is complete and minimal in space $L_{2}(0,1)$ because the boundary conditions (17)-(18) are regular by Birkhoff (see [11]).

We show that the systems of functions $V\left(B_{j, k}\right)$ and $V\left(L_{1, j, k}\right)$ are quadratically approximate in space $L_{2}(0,1)$.

Let us estimate the sum of the series

$$
\begin{aligned}
H\left(L_{1, j, k} ; B_{j, k}\right) & =\sum_{s, m}\left\|v_{s, m}\left(x_{2}, L_{1, j, k}\right)-v_{s, m}\left(x_{2}, B_{j, k}\right)\right\|_{L_{2}(0,1)}^{2} \\
& =\sum_{m=1}^{\infty}\left\|v_{0, m}\left(x_{2}, L_{1, j, k}\right)-v_{0, m}\left(x_{2}, B_{j, k}\right)\right\|_{L_{2}(0,1)^{\prime}}^{2} \\
H\left(L_{1, j, k}, B_{j, k}\right) & \leq \max \left|\eta_{j, m, k}\right|^{2}\left|\Delta_{j, 1, m, k}^{-2}\right| \sum_{m} \sum_{q=2}^{n}\left|\Delta_{j, q, m, k}\right|^{2}\left\|z_{1, q, m, k}\left(x_{2}\right)\right\|_{L_{2}(0,1)}^{2} .
\end{aligned}
$$


Taking into account the choice of numbers $\omega_{r, m, k}$, we obtain the estimate

$$
H\left(L_{1, j, k}, B_{j, k}\right)<\infty
$$

Therefore, the complete and minimal system $V\left(L_{1, j, k}\right) \in L_{2}(0,1)$ is quadratically approximate to Riesz basis $V\left(B_{1, k}\right)$.

Thus, applying the Bari theorem (see [10]), we obtain the statement of Lemma 6.

\section{TRANSFORMATION OPERATORS}

Let us determine

$$
z_{2, j, m, k}\left(x_{2}\right):=\eta_{j, m, k} z_{1, j, m, k}\left(x_{2}\right), \quad m=1,2, \ldots
$$

By choosing of arbitrary sequence of real numbers $\theta=\left\{\theta_{m}\right\}_{m=1}^{\infty}$ we define the operator $B_{j, \theta}: L_{2}(0,1) \rightarrow L_{2}(0,1)$, which is generated by the differential expression

$$
\sum_{p=0}^{n}(-1)^{p} a_{p} \mu_{k, 1}^{p} z^{(2 n-2 p)}\left(x_{2}\right)
$$

and has the system $V\left(B_{j, \theta}\right):=\left\{v_{s, m}\left(x_{2}, B_{j, \theta}\right) \in L_{2}(0,1): s=0,1, m=1,2, \ldots\right\}$ of functions

$$
\begin{aligned}
& v_{1, m}\left(x_{2}, B_{j, \theta}\right):=\tau_{1, m, 2}\left(x_{2}\right), \\
& v_{0, m}\left(x_{2}, B_{j, \theta}\right):=\tau_{0, m, 2}\left(x_{2}\right)+\theta_{m} z_{2, j, m, k}\left(x_{2}\right), m=1,2, \ldots,
\end{aligned}
$$

which are root functions in the sense of equalities

$$
\begin{aligned}
& B_{j, \theta} v_{1, m}\left(x_{2}, B_{j, \theta}\right)=\lambda_{k, m} v_{1, m}\left(x_{2}, B_{j, \theta}\right), m=1,2, \ldots, \\
& B_{j, \theta} v_{0, m}\left(x_{2}, B_{j, \theta}\right)=\lambda_{k, m} v_{0, m}\left(x_{2}, B_{j, \theta}\right)+\xi_{j, k, m} v_{1, m}\left(x_{2}, B_{j, \theta}\right),
\end{aligned}
$$

where $\xi_{j, k, m}=(-1)^{n} 4 n \eta_{j, m, k} \theta_{m} \sum_{p=0}^{n} a_{p} C_{2 n}^{2 n-2 p} \mu_{k, 1}^{p} \rho_{m, 2}^{2 n-2 p-1}, m=1,2, \ldots$, and has the set of eigenvalues $\sigma_{k}$.

Let us consider the operators $R\left(B_{j, \theta}\right)$, which are defined in the space $L_{2}(0,1)$ by

$$
\begin{aligned}
R\left(B_{j, \theta}\right) & :=E+S\left(B_{j, \theta}\right), \\
S\left(B_{j, \theta}\right) \tau_{1, m, 2}\left(x_{2}\right) & :=0, S\left(B_{j, \theta}\right) \tau_{0, m, 2}\left(x_{2}\right):=\theta_{m} z_{2, j, m, k}\left(x_{2}\right), \quad m=1,2, \ldots
\end{aligned}
$$

Let $Q_{j}\left(L_{0, k}\right)$ be the set of operators $B_{j, \theta}$, which have purely point spectrum $\sigma_{k}$ and the system of root functions (26), $\Gamma_{j}\left(L_{0, k}\right)$ be the set of operators $R\left(B_{j, \theta}\right)$.

For any $B_{j, \theta_{1}}, B_{j, \theta_{2}} \in \Gamma_{j}\left(L_{0, k}\right)$, we define on $\Gamma_{j}\left(L_{0, k}\right)$ the commutative multiplication operation

$$
R\left(B_{j, \theta_{1}}\right) R\left(B_{j, \theta_{2}}\right)=E+S\left(B_{j, \theta_{1}}\right)+S\left(B_{j, \theta_{2}}\right)=R\left(B_{j, \theta_{2}}\right) R\left(B_{j, \Theta_{1}}\right)
$$

and the inverse operator $R^{-1}\left(B_{j, \theta}\right)=E-S\left(B_{j, \theta}\right), B_{j, \theta} \in \Gamma_{1}\left(L_{0, k}\right)$.

Therefore, $\Gamma_{j}\left(L_{0, k}\right)$ is the Abelian group, which contains a subgroup $\Gamma_{j}\left(L_{0, k}\right) \cap\left[L_{2}(0,1)\right]$. 
Lemma 7. For any sequence $\left\{\theta_{m}\right\}_{m=1}^{\infty} \subset \mathbb{R}$ the system of functions $V\left(B_{j, \theta}\right)$ is complete and minimal in $L_{2}(0,1)$.

Proof. We prove on the contrary that the system of functions $V\left(B_{j, \theta}\right)$ is total (complete) in the space $L_{2}(0,1)$.

Let us suppose that there exists a function $h=h_{0}+h_{1}, h_{s} \in L_{s, 2}(0,1)$ that is orthogonal to all elements of the system $V\left(B_{j, \theta}\right)$. Taking into account, that the system $T_{1,2}$ is the orthonormal basis of space $L_{1,2}(0,1)$, we obtain $h_{1} \equiv 0$.

Therefore $h \in L_{0,2}(0,1)$. Assuming the orthogonality of the function $h$ to the elements of the system $V\left(B_{j, \theta}\right)$, we have equality

$$
\left(h, v_{0, m}\left(x_{2}, B_{j, \theta}\right)\right)_{L_{2}(0,1)}=\left(h, \tau_{0, m, 2}\right)_{L_{2}(0,1)}=0, m=1,2, \ldots .
$$

Taking into account that the system $T_{0,2}$ is the orthonormal basis of $L_{0,2}(0,1)$, we obtain $h \equiv 0$.

Let us prove the minimality of the system $V\left(B_{j, \theta}\right)$. We determine the set of functions

$$
L_{2, \theta}(0,1):=\left\{h=\sum_{r=0}^{1} \sum_{m=1}^{\infty} h_{r, m} \tau_{r, m, 2}\left(x_{2}\right) \in L_{2}(0,1): \sum_{r=0}^{1} \sum_{m=1}^{\infty} h_{r, m}^{2} \theta_{m, 0}^{2}<\infty\right\},
$$

where $\theta_{m, 0}:=1$, in the case $\theta_{m}=0, \theta_{m, 0}=\theta_{m}$, if $\theta_{m} \neq 0, m=1,2, \ldots$.

The set $L_{2, \theta}(0,1)$ is a Hilbert space with respect to the scalar product

$$
(h ; g)_{L_{2, \theta}(0,1)}:=\sum_{r=0}^{1} \sum_{m=1}^{\infty} \theta_{m, 0}^{2} h_{r, m} g_{r, m} .
$$

Let us consider the relations

$$
\begin{aligned}
v_{0, m}\left(x_{2}, B_{j, \theta}\right) & =R\left(B_{j, \theta}\right) \tau_{0, m, 2}\left(x_{2}\right)=\left(1-\theta_{m}\right) \tau_{0, m, 2}\left(x_{2}\right)+\theta_{m} v_{0, m}\left(x_{2}, L_{1, j, k}\right), \\
\left(h ; R\left(B_{j, \theta}\right) \tau_{0, m, 2}\right)_{L_{2}(0,1)}^{2} & \leq 4\left(1+\theta_{m}^{2}\right)\left(h ; \tau_{0, m, 2}\right)_{L_{2}(0,1)}^{2}+2 \theta_{m}^{2}\left(h ; v_{0, m}\left(x_{2}, L_{1, j, k}\right)\right)_{L_{2}(0,1)}^{2} \\
\left(h ; v_{0, m}\left(x_{2}, B_{j, \theta}\right)\right)_{L_{2}(0,1)} & =\left(R^{*}\left(B_{j, \theta}\right) h ; \tau_{0, m, 2}\right)_{L_{2}(0,1)}, \\
\left(h ; R\left(B_{j, \theta}\right) \tau_{1, m, 2}\right)_{L_{2}(0,1)} & =\left(h ; \tau_{1, m, 2}\right)_{L_{2}(0,1)}, m=1,2, \ldots
\end{aligned}
$$

Taking into account these relations and inequality

$$
\left(h ; v_{s, m}\left(x_{2}, L_{1, j, k}\right)\right)_{L_{2}(0,1)}^{2} \leq\left\|R^{*}\left(L_{1, j, k}\right)\right\|_{\left[L_{2}(0,1)\right]}^{2}\left(h ; \tau_{s, m, 2}\right)_{L_{2}(0,1)}^{2}, s=0,1, m=1,2, \ldots,
$$

we obtain the estimate

$$
\left\|R^{*}\left(B_{j, \theta}\right) h\right\|_{L_{2}(0,1)}^{2} \leq\left(4+\left\|R^{*}\left(L_{1, j, k}\right)\right\|_{\left[L_{2}(0,1)\right]}^{2}\right)\|h\|_{L_{2, \theta}(0,1)}^{2} .
$$

Therefore, for conjugate operator $R^{*}\left(B_{j, \theta}\right)$ the following inclusion holds (see [9])

$$
R^{*}\left(B_{j, \theta}\right) \in\left[L_{2, \theta}(0,1) ; L_{2}(0,1)\right] .
$$

So, the inverse operator exists

$$
E-S^{*}\left(B_{j, \theta}\right) \in\left[L_{2, \theta}(0,1) ; L_{2}(0,1)\right]
$$

that is, the system of functions $V\left(L_{1, j, k}\right)$ has the unique biorthogonal system $W\left(L_{1, j, k}\right)$. 
Lemma 8. The system of functions $V\left(B_{j, \theta}\right)$ is the Riesz basis in $L_{2}(0,1)$ if and only if the sequence $\left\{\theta_{m}\right\}_{m=1}^{\infty}$ is bounded.

Proof. Necessity. If the system of functions $V\left(B_{j, \theta}\right)$ is the Riesz basis, then it is almost normalized. From the opposite, if $\left|\theta_{m}\right| \rightarrow \infty$ for $m \rightarrow \infty$, then, taking into account (27)-(28), we obtain

$$
\left\|v_{0, m}\left(x_{2}, B_{j, \theta}\right)\right\|_{L_{2}(0,1)}=1+\left|\theta_{m}\right|\left\|z_{2, j, m, k}\right\|_{L_{2}(0,1)} \rightarrow \infty, m \rightarrow \infty .
$$

Sufficiency. If the sequence $\theta$ is bounded, then the spaces $L_{2, \theta}(0,1)$ and $L_{2}(0,1)$ coincide. Therefore, taking into account the inclusion (29), we obtain $R\left(B_{j, \theta}\right) \in\left[L_{2}(0,1)\right]$.

The set of $n$ real sequences $\left\{\theta_{j, m}\right\}_{m=1}^{\infty}, j=1,2, \ldots, n$, we denote by $\Theta$, and consider the operator $B_{\Theta}$, eigenvalues of which coincide with the eigenvalues of the operator $L_{0, k}$ and eigenfunctions are defined by the equalities

$$
\left\{\begin{array}{l}
v_{1, m}\left(x_{2}, B_{\Theta}\right)=\tau_{1, m, 2}\left(x_{2}\right), \\
v_{0, m}\left(x_{2}, B_{\Theta}\right)=\tau_{0, m, 2}\left(x_{2}\right)+\sum_{j=1}^{n} \theta_{j, m} z_{2, j, m, k}\left(x_{2}\right), m=1,2, \ldots
\end{array}\right.
$$

We define the transformation operator $R\left(B_{\Theta}\right):=E+S\left(B_{\Theta}\right): L_{2}(0,1) \rightarrow L_{2}(0,1)$ which maps the system of eigenfunctions $V\left(L_{0, k}\right)$ of operator $L_{0, k}$ into system of functions $V\left(B_{\Theta}\right)$ of operator $B_{\Theta}$

$$
R\left(B_{\Theta}\right) \tau_{s, m, 2}\left(x_{2}\right):=v_{s, m, k}\left(t, B_{\Theta}\right), s=0,1, m=1,2, \ldots
$$

From the definition of operator $B_{\Theta}$ we obtain

$$
S\left(B_{\Theta}\right): L_{0,2}(0,1) \rightarrow L_{1,2}(0,1), \quad L_{1,2}(0,1) \rightarrow 0, \quad S^{2}\left(B_{\Theta}\right)=0 .
$$

Therefore, the bounded operator $R^{-1}\left(B_{\Theta}\right)=E-S\left(B_{\Theta}\right)$ exists.

Lemma 9. For any sequences $\left\{\theta_{j, m}\right\}_{m=1}^{\infty}, j=1,2, \ldots, n$, the system of eigenfunctions of operator $B_{\Theta}$ is complete and minimal in the space $L_{2}(0,1)$.

The system of functions $V\left(B_{\Theta}\right)$ is the Riesz basis in the space $L_{2}(0,1)$ if and only if the sequences $\left\{\theta_{j, m}\right\}_{m=1}^{\infty}, j=1,2, \ldots, n$, are bounded.

Proof of Lemma 9 is similar to the proof of Lemma 7.

Let $Q\left(L_{k}\right)$ be the set of operators $B_{\Theta}$, eigenfunctions of which is defined by formulas (30), $\Gamma\left(L_{k}\right)$ be the set of transformation operators $R\left(B_{\Theta}\right)$.

Remark 3. On the set $\Gamma\left(L_{k}\right)$ we can define the multiplication operation and prove that $\Gamma\left(L_{k}\right)$ is an Abelian group.

\section{THE NONSELF-AJOINT PROBLEM FOR A DIFFERENTIAL EQUATION OF EVEN ORDER}

For equation (9) let us consider the eigenvalues problem with nonlocal conditions

$$
\left\{\begin{array}{l}
l_{2, s, 2} z:=z^{(2 s-2)}(0)+z^{(2 s-2)}(1)=0, s=1,2, \ldots, n, \\
l_{2, n+j, 2} z:=z^{(2 j-1)}(0)+z^{(2 j-1)}(1)+l_{n+j}^{2} z=0, \\
l_{2, n+s, 2} z:=z^{(2 j-1)}(0)+z^{(2 j-1)}(1)=0, s \neq j, s=1,2, \ldots, n,
\end{array}\right.
$$


where

$$
l_{n+j}^{2} z:=\sum_{q=0}^{k_{s, 2}} \sum_{r=0}^{k_{2}} b_{s, q, j, 2} z^{(q)}\left(x_{2, r}\right) .
$$

Let $L_{2, j, k}: L_{2}(0,1) \rightarrow L_{2}(0,1)$ be the operator of the problem (9), (31), (32)

$$
\begin{aligned}
L_{2, j, k} z\left(x_{2}\right) & :=\sum_{p=0}^{n}(-1)^{p} a_{p} \mu_{k, 1}^{p} z^{(2 n-2 p)}\left(x_{2}\right), z \in D\left(L_{2, j, k}\right), \\
D\left(L_{2, j, k}\right) & :=\left\{z \in W_{2}^{2 n}(0,1): \ell_{2, r, 2} z=0, r=1,2, \ldots, 2 n\right\},
\end{aligned}
$$

and $V\left(L_{2, j, k}\right)$ be the system of root functions for operator $L_{2, j, k}$

$$
R\left(L_{2, j, k}\right): L_{2}(0,1) \rightarrow L_{2}(0,1), \quad R\left(L_{2, j, k}\right): V\left(L_{0, k}\right) \rightarrow V\left(L_{2, j, k}\right) .
$$

Lemma 10. Let Assumption $P_{1}$ holds. Then the operator $L_{2, j, k}$ has the system of root functions $V\left(L_{2, j, k}\right)$, which is complete and minimal in the space $L_{2}(0,1)$.

If Assumptions $P_{1}, P_{3}$ hold, then the system of functions $V\left(L_{2, j, k}\right)$ is the Riesz basis in the space $L_{2}(0,1)$.

Proof. Substituting function $\tau_{1, m, 2}\left(x_{2}\right)$ into boundary conditions (31), (32) we obtain that the operator $L_{2, j, k}$ has eigenvalues

$$
v_{1, m}\left(x_{2}, L_{2, j, k}\right):=\tau_{1, m, 2}\left(x_{2}\right), m=1,2, \ldots .
$$

Root function $v_{0, m}\left(x_{2}, L_{2, j, k}\right)$ of operator $L_{2, j, k}$ is defined by the sum

$$
v_{0, m}\left(x_{2}, L_{2, j, k}\right):=\tau_{0, m, 2}\left(x_{2}\right)+\eta_{j, m, k}^{1} z_{2, j, m, k}\left(x_{2}\right), m=1,2, \ldots
$$

For determining of unknown parameters $\eta_{j, m, k}^{1}$ we substitute the expression (34) into boundary conditions (31), (32).

Taking into account the ratio (22) we have the equality

$$
\eta_{j, m, k}^{1}=-\left(l_{2, n+j, 2} z_{2, j, m, k}\right)^{-1} l_{n+j}^{2} \tau_{0, m, 2} \text {. }
$$

Therefore, the operator $L_{2, j, k}$ has the system of root functions (33)-(35).

Remark 4. On the contrary, as in the proof of Lemma 8, we can prove the completeness of the system $V\left(L_{2, j, k}\right)$ in the space $L_{2}(0,1)$.

Taking into account that $z_{2, j, m, k}\left(x_{2}\right) \in L_{1,2}(0,1)$, we have the inclusion $R\left(L_{2, j, k}\right) \in \Gamma\left(L_{0, k}\right)$. Therefore, the system $V\left(L_{2, j, k}\right)$ is minimal in the space $L_{2}(0,1)$.

Let Assumption $P_{3}$ holds. Then from the inequality (25) we obtain $\left|\eta_{j, m, k}^{1}\right| \leq C_{8}$. Therefore, taking into account the statement of Lemma 9, we obtain that $R\left(L_{2, j, k}\right) \in \Gamma\left(L_{0, k}\right) \cap\left[L_{2}(0,1)\right]$.

Let us show that for the operator $R\left(L_{k}\right)$ Lemma 10 holds. Substituting into boundary conditions (31), (32) we obtain that the operator $L_{k}$ has the eigenfunctions

$$
v_{1, m}\left(x_{2}, L_{k}\right):=\tau_{1, m, 2}\left(x_{2}\right), m=1,2, \ldots
$$

Root function $v_{0, m}\left(x_{2}, L_{k}\right)$ of operator $L_{k}$ is defined by the sum

$$
v_{0, m}\left(x_{2}, L_{k}\right):=\tau_{0, m, 2}\left(x_{2}\right)+\sum_{j=1}^{n} \eta_{j, m, k}^{1} z_{2, j, m, k}\left(x_{2}\right), m=1,2, \ldots
$$


where unknown parameters $\eta_{j, m, k}^{1}$ are defined by formula (35).

Therefore, the transformation operator $R\left(L_{k}\right): L_{2}(0,1) \rightarrow L_{2}(0,1)$

$$
R\left(L_{k}\right) \tau_{s, m, 2}\left(x_{2}\right):=v_{s, m}\left(x_{2}, L_{k}\right), s=0,1, m=1,2, \ldots,
$$

is the element of the set $\Gamma\left(L_{k}\right)$. Thus, the system $V\left(L_{k}\right)$ is complete and minimal in the space $L_{2}(0,1)$.

Taking into account the ratio $R\left(L_{k}\right)=\prod_{j=1}^{n} R\left(L_{2, j, k}\right)$ and the statement of Lemma 9, in the case of Assumptions $P_{1}, P_{3}$ we have $R\left(L_{k}\right) \in\left[L_{2}(0,1)\right]$.

Thus, the system $V\left(L_{k}\right)$ is the Riesz basis in the space $L_{2}(0,1)$.

Therefore, for operator $L_{k}$ Lemma 10 holds.

\section{PROOF OF THE MAIN RESULTS}

Proof of Theorem 1. Particular cases of the operator $L$, when $b_{q, r, s, j}=0$, we denoted by $L^{j}, j=1,2$, respectively.

Let $\pi_{r, k, 1}$ be the orthoprojector into one-dimensional proper subspace in $L_{2}(0,1)$. We define the root functions of operator $L^{2}$ by

$$
v_{s, r, k, m}\left(x, L^{2}\right):=v_{s, m}\left(x_{2}, L_{k}\right) \tau_{r, k, 1}\left(x_{1}\right), s, r \in\{0,1\}, k, m \in \mathbb{N},
$$

and the transformation operator $R\left(L^{2}\right): L_{2}(G) \rightarrow L_{2}(G)$ by

$$
\begin{aligned}
R\left(L^{2}\right) & :=\sum_{r, k, m} R\left(L_{k}\right) \times \pi_{r, k, 1} \\
v_{s, r, k, m}\left(x, L^{2}\right) & :=R\left(L^{2}\right) v_{s, r, k, m}\left(x, L_{0}\right), s, r \in\{0,1\}, k, m \in \mathbb{N} .
\end{aligned}
$$

Similarly, when Assumption $P_{2}$ holds, we can define the biorthogonal system $W\left(L^{2}\right)$.

Taking into account Lemma 10, we obtain that for operator $L^{2}$ Theorem 1 holds. From Theorem 1 of the paper [1] we obtain that for operator $L^{1}$ Theorem 1 holds and for transformation operator $R\left(L^{1}\right)$ the ratio $R\left(L^{1}\right) \in\left[L_{2}(G)\right]$ holds.

Let us define the transformation operator $R(L): L_{2}(G) \rightarrow L_{2}(G), R(L):=R\left(L^{1}\right) R\left(L^{2}\right)$, and the root functions of operator $L$

$$
v_{s, r, k, m}(x, L):=R(L) v_{s, r, k, m}\left(x, L_{0}\right), s, r \in\{0,1\}, k, m \in \mathbb{N} .
$$

By direct verification we obtain that the elements of the system $V(L)$ are roots in sense

$$
\begin{aligned}
\left(L-\lambda_{k, m}\right) v_{r, 1, k, m}(x, L) & =0, \\
\left(L-\lambda_{k, m}\right) v_{r, 0, k, m}(x, L) & =\xi_{r, 0, k, m} v_{r, 1, k, m}(x, L), \\
\xi_{r, 0, k, m} & =4 n(-1)^{n-1} \eta_{r, 0, k, m} \rho_{m, 2}^{2 n-1}, \quad r, s \in\{0,1\}, \quad k, m \in \mathbb{N} .
\end{aligned}
$$

Taking into account Assumption $P_{1}$, we obtain that the systems $V\left(L^{j}\right)$ have the unique biorthogonal systems $W\left(L^{j}\right), j=1,2$, in the space $L_{2}(G)$.

Therefore, the system $V(L)$ is complete and minimal in the space $L_{2}(G)$.

Let Assumptions $P_{1}-P_{3}$ hold. Then from Theorem 3 of the paper [8] and from Lemma 10 we obtain $R\left(L^{1}\right) \in\left[W_{2}^{2 n}(G)\right]$. So, Theorem 1 is proved. 
Remark 5. There are positive numbers $C_{9}, C_{10}$ such that for any function

$$
f(x)=\sum_{r, q, k, m} f_{r, q, k, m} v_{r, q, k, m}(x, L)
$$

the inequality

$$
C_{9}\|f\|_{L_{2}(G)}^{2} \leq \sum_{r, q, k, m}\left|f_{r, q, k, m}\right|^{2} \leq C_{10}\|f\|_{L_{2}(G)}^{2}
$$

holds.

Proof of Theorem 2. It is enough to consider the case $q_{q, r, s, 1}=0$. Let the right part of the equation (1) has the expansion (36).

The solution of the problem (1)-(2) we find in the form of a series

$$
u(x)=\sum_{r, q, k, m} u_{r, q, k, m} v_{r, q, k, m}(x, L) .
$$

Substituting these expansions into equation (1) we obtain the equalities

$$
\begin{aligned}
& u_{r, 0, k, m}=\lambda_{k, m}^{-1} f_{r, 0, k, m} \\
& u_{r, 1, k, m}=\lambda_{k, m}^{-1} f_{r, 1, k, m}-\lambda_{k, m}^{-2} \xi_{r, 0, k, m} f_{r, 0, k, m}, r \in\{0,1\}, k, m \in \mathbb{N} .
\end{aligned}
$$

Let us consider the relations

$$
D_{1}^{2 n} u(x)=\sum_{r, q, k, m} \lambda_{k, m}^{-1} \mu_{k, 1}^{n} f_{r, q, k, m} v_{r, q, k, m}(x, L) .
$$

Taking into account Assumption $P_{2}$, we obtain

$$
\left\|D_{1}^{2 n} u\right\|_{L_{2}(G)} \leq C_{11}\|f\|_{L_{2}(G)} .
$$

Similarly from Theorem 3 of the paper [1] we obtain the inequality

$$
\left\|D_{2}^{2 n} u\right\|_{L_{2}(G)} \leq C_{12}\|f\|_{L_{2}(G)} .
$$

Therefore, taking into account Theorem 3, we obtain $R\left(L^{2}\right) \in\left[W_{2}^{2 n}(G)\right]$.

Thus, for the definition of the transformation operator $R(L)$ we have $R(L) \in\left[W_{2}^{2 n}(G)\right]$. Then

$$
\|u\|_{W_{2}^{2 n}(G)} \leq C_{13}\|f\|_{L_{2}(G)} .
$$

Theorem is proved.

\section{REFERENCES}

[1] Baranetskij Ya.O., Ivasiuk I.Ya., Kalenyuk P.I., Solomko A.V. The nonlocal boundary problem with perturbations of antiperiodicity conditions for the elliptic equation with constant coefficients. Carpathian Math. Publ. 2018,10 (2), 215-234. doi:10.15330/cmp.10.2.215-234

[2] Baranetskij Y.O., Kalenyuk P.I. Boundary-value problems with Birkhoff regular but not strongly regular conditions for a second-order differential operator. J. Math. Sci. 2019, 238 (1), 1-21. doi:10.1007/s10958-019-04214-Z

[3] Baranetskij Ya.O., Kalenyuk P.I., Kolyasa L.I. Spectral properties of nonself-adjoint nonlocal boundary-value problems for the operator of differentiation of even order. Ukr. Math. J. 2018, 70 (6), 851-865. doi:10.1007/s11253-0181538-4 
[4] Baranetskij Ya.O., Kalenyuk P.I. Nonlocal multipoint problem with multiple spectrum for an ordinary (2n)th order differential equation. J. Math. Sci. 2020, 246 (2), 152-169. doi:10.1007/s10958-020-04727-y

[5] Baranetskij Ya.O., Kalenyuk P.I., Kolyasa L.I. Nonlocal multipoint problem for an ordinary differential equations of even order involution. Mat. Stud. 2018, 49 (1), 80-94. doi:10.15330/ms.49.1.80-94

[6] Baranetskij Ya.O., Kalenyuk P.I., Kolyasa L.I., Kopach M.I. The nonlocal problem for the differential-operator equation of the even order with the involution. Carpathian Math. Publ. 2017, 9 (2), 109-119. doi:10.15330/cmp.9.2.109119

[7] Baranetskij Ya.O., Demkiv I.I., Ivasiuk I.Ya., Kopach M.I. The nonlocal problem for the differential equations the order $2 n$ with an unbounded operator coefficients with the involution. Carpathian Math. Publ. 2018, 10 (1), 14-30. doi:10.15330/cmp.10.1.14-30

[8] Baranetskij Ya.O., Kalenyuk P.I., Kopach M.I., Solomko A.V. The nonlocal boundary value problem with perturbations of mixed boundary conditions for an elliptic equation with constant coefficients. I. Carpathian Math. Publ. 2019, 11 (2), 228-239. doi:10.15330/cmp.11.2.228-239

[9] Berezanskii M.M. Expansion in eigenfunctions of self-adjoint operators. American Math. Soc., 1968.

[10] Gokhberg I.Ts., Krein M.G. Introduction to the theory of linear non self-adjoint operators. Nauka, Moscow, 1965. (in Russian)

[11] Naimark M.A. Linear differential operators. Frederick Ungar Publ. Co., New York, 1967.

[12] Katrakhov V.V., Sitnik S.M. The method of transformation operators and the boundary value problems for singular elliptic equations. Sovremennaya matematika. Fundamentalnye napravleniya 2018, 64 (2), 211-426. (in Russian)

Received 25.03.2020

Revised 21.05.2020

Баранецький Я.О., Каленюк П.І., Копач М.І., Соломко А.В. Нелокальна крайова задача зі збуреннями мішаних крайових умов для еліптичного рівняння із сталими коефічієнтами. II // Карпатські матем. публ. - 2020. - Т.12, №1. - С. 173-188.

У роботі продовжено розпочаті у першій частині дослідження властивостей задачі з нелокальними умовами, які є багатоточковими збуреннями мішаних крайових умов. Зокрема, побудовано узагальнений оператор перетворення, який відображає розв' язки самоспряженої крайової задачі із мішаними крайовими умовами в розв'язки багатоточкової задачі. Побудовано систему $V(L)$ кореневих функцій оператора $L$ багатоточкової задачі. Визначено умови, при яких система $V(L)$ повна та мінімальна та умови, за яких вона є базисом Piсcа. $\Delta \wedge я$ випадку еліптичного рівняння встановлено умови існування та єлиності розв' язку задачі.

Ключові слова і фрази: диференціальне рівняння з частинними похідними, кореневі функції, метод Фур'є, метод операторів перетворення, базис Рісса. 